\title{
Diffusion Kurtosis Imaging Detects Microstructural Changes in the Brain after Acute Alcohol Intoxication in Rats
}

\author{
Xi-ran Chen, Jie-ying Zeng, Zhi-Wei Shen, Ling-mei Kong, and Wen-bin Zheng \\ Department of Radiology, The Second Affiliated Hospital, Shantou University Medical College, Shantou, Guangdong, China
}

Correspondence should be addressed to Wen-bin Zheng; hwenb@126.com

Received 5 September 2016; Revised 26 November 2016; Accepted 12 December 2016; Published 18 January 2017

Academic Editor: Jiun-Jie Wang

Copyright (C) 2017 Xi-ran Chen et al. This is an open access article distributed under the Creative Commons Attribution License, which permits unrestricted use, distribution, and reproduction in any medium, provided the original work is properly cited.

The aim of this study was to test the technical feasibility of diffusion kurtosis imaging (DKI) in the brain after acute alcohol intoxication. Diffusion tensor imaging (DTI) and DKI during 7.0 T MRI were performed in the frontal lobe and thalamus before and $30 \mathrm{~min}, 2 \mathrm{~h}$, and $6 \mathrm{~h}$ after ethyl alcohol administration. Compared with controls, mean kurtosis values of the frontal lobe and thalamus first decreased by $44 \%$ and $38 \%$ within $30 \mathrm{~min}$ ( $p<0.01$ all) and then increased by $14 \%$ and $46 \%$ at $2 \mathrm{~h}$ (frontal lobe, $p>0.05$; thalamus, $p<0.01$ ) and by $29 \%$ and $68 \%$ at 6 h (frontal lobe, $p<0.05$; thalamus, $p<0.01$ ) after acute intake. Mean diffusivity decreased significantly in both the frontal lobe and the thalamus at various stages. However, fractional anisotropy decreased only in the frontal lobe, with no detectable change in the thalamus. This demonstrates that DKI possesses sufficient sensitivity for tracking pathophysiological changes at various stages associated with acute alcohol intoxication and may provide additional information that may be missed by conventional DTI parameters.

\section{Introduction}

Acute alcohol exposure may directly or indirectly affect the brain given many neurological changes such as blood flow, electrical brain activity, and memory [1-3]. Early medical and rehabilitative intervention may reduce more severe secondary damage to the brain, but both CT and conventional MRI usually fail to detect macroscopic and microscopic evidence of brain injury in acute alcohol exposure [4]. Thus, there is much interest in developing advanced neuroimaging techniques to detect and characterize microstructural changes that occur in the brain due to acute alcohol exposure [5-7].

In our previous study, we showed that diffusion tensor imaging (DTI) can detect microscopic tissue damage in the brain after acute alcohol intoxication based on fractional anisotropy (FA) and apparent diffusion coefficient values $[8,9]$. DTI measurements are based on the assumption of a Gaussian displacement probability distribution of water molecules due to water self-diffusion, such as water in isotropic liquid media [10]. However, diffusion of water molecules in most biological tissues, especially brain tissues, is restricted by barriers, such as cellular membranes, which cause diffusion to deviate substantially from a Gaussian form [11, 12], making DTI a limited indicator of complexity.
Therefore, new imaging methods based on non-Gaussian diffusion models, such as diffusion kurtosis imaging (DKI), may assess microstructural complexity more accurately than DTI, especially in gray matter [13-15]. Moreover, DKI datasets generally include DTI datasets as part of the total measurements [16].

The aims of this study were as follows: (1) to investigate microstructural abnormalities in the frontal lobe and thalamus of rat brains after acute alcohol intoxication using DKI metrics and DTI metrics, (2) to determine whether DKI provides additional information about underlying structural abnormalities compared with DTI, and (3) to investigate the kinetics of structural and functional changes in the brain at various stages following alcohol administration.

\section{Materials and Methods}

2.1. Animal Models. Thirty-five sexually mature (8- to 10 week-old) male Sprague-Dawley rats (200-250 g, from the Laboratory Animal Center of Shantou University Medical College, Shantou, Guangdong, China) were divided into 4 groups: 3 acute ethyl alcohol (EtOH) intoxication groups ( $30 \mathrm{~min}, 2 \mathrm{~h}$, and $6 \mathrm{~h} ; n=10$ for each group) and 1 control group $(n=5)$. Rats in the acute EtOH intoxication groups 


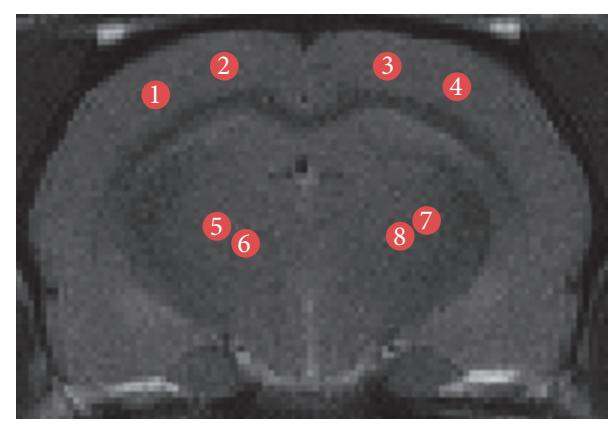

(a)



(b)

FIGURE 1: Illustration of ROIs on T2WI (a) and MK (b) maps for a representative rat on coronal slices. Regions shown are frontal lobe (1-4) and thalamus (5-8).

received an intragastrically injection of EtOH (Hongxing Erguotou wine, $56 \%$ vol, Beijing, China) at a dose of $15 \mathrm{~mL} / \mathrm{kg}$ and were then examined every minute after EtOH administration for loss of the righting reflex to ensure acute alcohol intoxication [17]. The control group received $15 \mathrm{~mL} / \mathrm{kg}$ of saline by intragastrically injection before MRI examination. The study protocol was approved by the ethics committee of Shantou University Medical College.

2.2. Imaging. All rats were scanned in a 7.0 T (Agilent VnmrJ 3 Imaging, USA) animal MR machine using a surface coil with a diameter of $30 \mathrm{~mm}$. Rats in the 3 subgroups of acute EtOH intoxication were imaged separately at $30 \mathrm{~min}$, $2 \mathrm{~h}$, and $6 \mathrm{~h}$ after EtOH administration. The animals were anesthetized with $4 \%$ isoflurane mixed with $1 \mathrm{~L} / \mathrm{min}$ oxygen and maintained with $2 \%$ isoflurane.

A 3-slice (axial, sagittal, and coronal) scout was used to ensure that the brain was in the proper position. T2-weighted imaging was obtained using $2 \mathrm{D}$ rapid acquisition with a fast spin-echo sequence using a $2000 \mathrm{~ms}$ repetition time (TR) and 5 slices at $2 \mathrm{~mm}$ thickness with a 0.2 gap and an in-plane resolution of $128 \times 96 \mathrm{um}$. The T2-weighted imaging was used to position the DKI field of view.

Diffusion kurtosis imaging was obtained with a fast spin-echo multislice sequence, together with an in-plane resolution of $280 \times 260 \mathrm{um}$ and a TR/TE of 2000/36 ms at $2 \mathrm{~mm}$ thickness with a 0.2 gap, 2 averages, 4 shots, and 16 kzero. Two $b$-values $\left(1000 \mathrm{~s} / \mathrm{mm}^{2}\right.$ and $2000 \mathrm{~s} / \mathrm{mm}^{2}$ ) were applied to 30 directions following acquisition of the image at $b=0 \mathrm{~s} / \mathrm{mm}^{2}$. Scan time for DKI was approximately $33 \mathrm{~min}$.

Shimming was very important to MR image quality. Therefore, we only selected images that achieved a line width of less than $20 \mathrm{~Hz}$ in repeated shimming attempts.

2.3. Histology. After all images were obtained, animals were immediately killed for histology. Animals received an overdose of chloral hydrate and were perfused transcardially with $4 \%$ paraformaldehyde in $0.1 \mathrm{M}$ phosphate buffer. Brains were removed and fixed with $4 \%$ paraformaldehyde for $48 \mathrm{~h}$. After fixation, brains were embedded in paraffin, and contiguous $5 \mu \mathrm{m}$ sections at the level of the frontal lobe and thalamus were cut on a microtome (Rm 2016, LEICA, Germany). Sections were then stained with hematoxylin and eosin (HE).
2.4. Data Processing. For image analysis, MK, FA, and MD maps were processed with MATLAB (The Mathworks, Natick, MA, USA) and MRIcro software (Neuropsychology Lab, Columbia, SC, USA). Besides, because DKI datasets generally include DTI datasets as part of the total measurements, the FA and MD values were calculated by using part of the DKI model in equation (using only $b$-value $=1,000 \mathrm{~s} / \mathrm{mm}^{2}$ ) $[18,19]$.

Regions of interest (ROIs) included the thalamus and frontal lobe (including precentral area and striate cortex). There were two reasons that we chose frontal lobe and thalamus as our regions of interest. On the one hand, because of the high sensitivity of 7.0 $\mathrm{T}$ animal MR machine and the high requirement about the shimming, we just chose one slice. On the other hand, these areas are more vulnerable to acute alcohol intoxication [8]. Frontal lobe plays a major role in cognitive functions such as working memory, decision-making, motor controlling, and language [20]. As the central relay station of the brain, the thalamus has mutual projections to the cerebral cortex, which also has a vital role in neuropsychological performance such as attention, concentration, and information processing [21, 22]. Damage to the frontal lobe and thalamus is associated with clinical sequelae and cognitive impairment.

The average MK, FA, and MD values were calculated bilaterally on the left and right sides in the ROIs (Figure 1). Every ROI was tested four times to obtain the mean value and every region of interest was about $5 \mathrm{~mm}^{2}$.

2.5. Statistical Analysis. Each of the measured parameters $(\mathrm{MD}, \mathrm{FA}$, and $\mathrm{MK})$ was expressed as mean \pm standard deviation. Data analysis was conducted using SPSS 17.0 (SPSS Inc., Chicago, IL, USA) with a one-way ANOVA to compare groups. A level of $p<0.05$ was considered statistically significant.

\section{Results}

No obvious signal intensity abnormalities were observed in the T2-weighted images of all rats, whereas differences were detected in $\mathrm{MK}, \mathrm{MD}$, and FA parameters in the frontal lobe and thalamus (Figure 2). Comparisons of $\mathrm{MK}, \mathrm{MD}$, and FA values in different ROIs obtained from pre- and postalcohol administration are reported in Figure 3 and Table 1. 


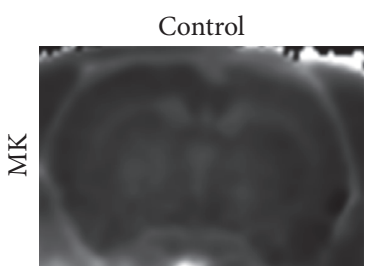

(a)

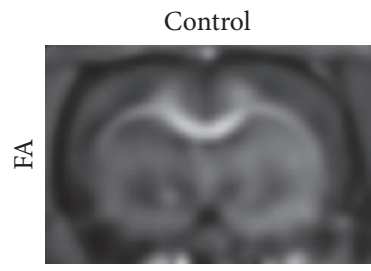

(e)

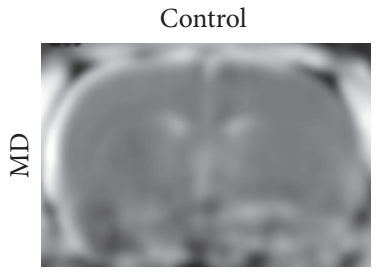

(i)

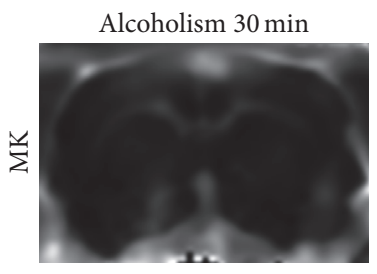

(b)

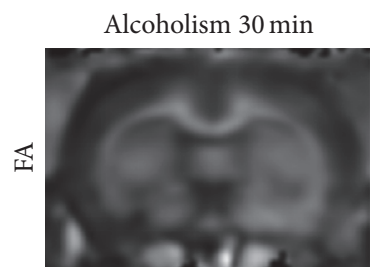

(f)

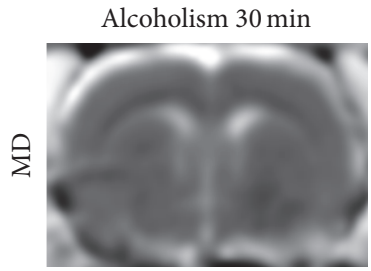

(j)

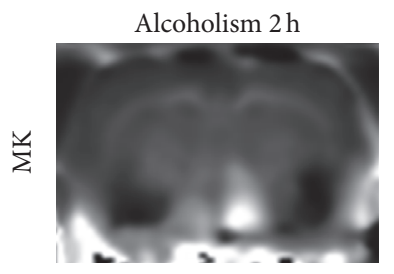

(c)

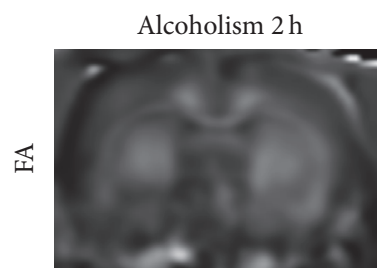

(g)

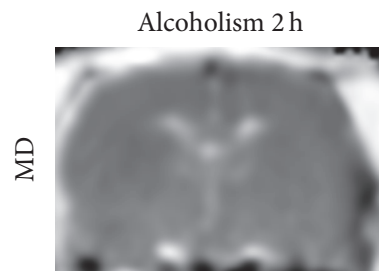

$(\mathrm{k})$

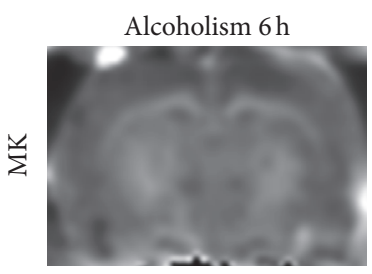

(d)

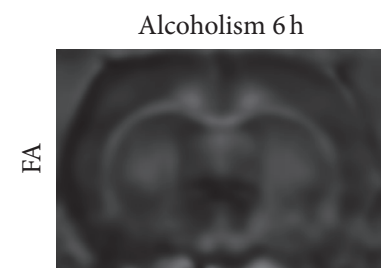

(h)

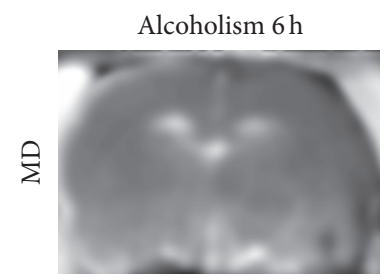

(1)

FIGURE 2: Representative MK, FA, and MD maps from controls and EtOH-treated rats. (a-d) MK values in the control group and in rats with alcohol intoxication at $30 \mathrm{~min}, 2 \mathrm{~h}$, and $6 \mathrm{~h}$; (e-h) FA values in the control group and in rats with alcohol intoxication at $30 \mathrm{~min}, 2 \mathrm{~h}$, and $6 \mathrm{~h}$; (i-l) MD values in the control group and in rats with alcohol intoxication at $30 \mathrm{~min}, 2 \mathrm{~h}$, and $6 \mathrm{~h}$.

TABLE 1: Comparisons of regional DKI and DTI indices, in the frontal lobe and thalamus, between controls and EtOH-administered rats $($ mean $\pm \mathrm{SD})$.

\begin{tabular}{|c|c|c|c|c|}
\hline DKI values & $\begin{array}{l}\text { Controls } \\
(N=5)\end{array}$ & $\begin{array}{l}\text { Alcoholism } 30 \mathrm{~min} \\
\qquad(N=10)\end{array}$ & $\begin{array}{l}\text { Alcoholism } 2 \mathrm{~h} \\
\quad(N=10)\end{array}$ & $\begin{array}{l}\text { Alcoholism 6h } \\
\qquad(N=10)\end{array}$ \\
\hline \multicolumn{5}{|l|}{ Frontal lobe } \\
\hline MK & $0.672 \pm 0.181$ & $0.375 \pm 0.137^{* *}$ & $0.765 \pm 0.083^{\Delta \Delta}$ & $0.869 \pm 0.065^{* * \Delta \Delta}$ \\
\hline FA & $0.257 \pm 0.129$ & $0.192 \pm 0.042^{*}$ & $0.234 \pm 0.077$ & $0.194 \pm 0.027$ \\
\hline $\mathrm{MD}$ & $0.800 \pm 0.086$ & $0.650 \pm 0.046^{* *}$ & $0.680 \pm 0.092^{* *}$ & $0.617 \pm 0.085^{* *}$ \\
\hline \multicolumn{5}{|l|}{ Thalamus } \\
\hline MK & $0.690 \pm 0.181$ & $0.425 \pm 0.191^{* *}$ & $1.010 \pm 0.187^{* * \Delta \Delta}$ & $1.161 \pm 0.125^{* * \Delta \Delta}$ \\
\hline FA & $0.397 \pm 0.123$ & $0.345 \pm 0.072$ & $0.417 \pm 0.070^{\Delta}$ & $0.390 \pm 0.069$ \\
\hline $\mathrm{MD}$ & $0.780 \pm 0.048$ & $0.623 \pm 0.088^{* *}$ & $0.689 \pm 0.095^{\Delta}$ & $0.628 \pm 0.071^{* *}$ \\
\hline
\end{tabular}

${ }^{* \Delta} p<0.05$ was considered to indicate a statistically significant difference.

${ }^{* * \Delta \Delta} p<0.01$ was considered to indicate a clear statistically significant difference.

* Statistical significance was based on comparison with controls.

${ }^{\Delta}$ Statistical significance was based on comparison with alcoholism $30 \mathrm{~min}$.

Compared with controls, MK decreased by $44 \%$ and $38 \%$ in the frontal lobe and thalamus at $30 \mathrm{~min}$ (frontal lobe: $p<0.01$; thalamus: $p<0.01$ ) and increased by $29 \%$ and $68 \%$ at 6 h (frontal lobe: $p<0.01$; thalamus: $p<0.01$ ). At $2 \mathrm{~h}$, the observed increase (14\% and $46 \%$ ) did not reach statistical significance in the frontal lobe (frontal lobe: $p>$ 0.05; thalamus: $p<0.01$ ). Compared with $30 \mathrm{~min}$ after acute alcohol administration, MK increased by $104 \%$ and $138 \%$ in the frontal lobe and thalamus at $2 \mathrm{~h}$ and increased by $132 \%$ and $173 \%$ at $6 \mathrm{~h}$ (all: $p<0.01$ ).
Compared with controls, a significant decrease in FA was observed in the frontal lobe at $30 \mathrm{~min}(p<0.05)$ after acute alcohol administration, but the trend in reduced FA at the $2 \mathrm{~h}$ and $6 \mathrm{~h}$ time points was not significant $(p>0.05)$. No statistically significant differences were observed for the FA values in the thalamus at any of the 3 time points $(p>0.05)$. MD showed decreases both in the frontal lobe and thalamus at each of the 3 time points except at $2 \mathrm{~h}$ in the thalamus $(2 \mathrm{~h}$ in the thalamus: $p>0.05$; others: $p<0.01$ ). Compared with 30 min after acute alcohol administration, a significant 

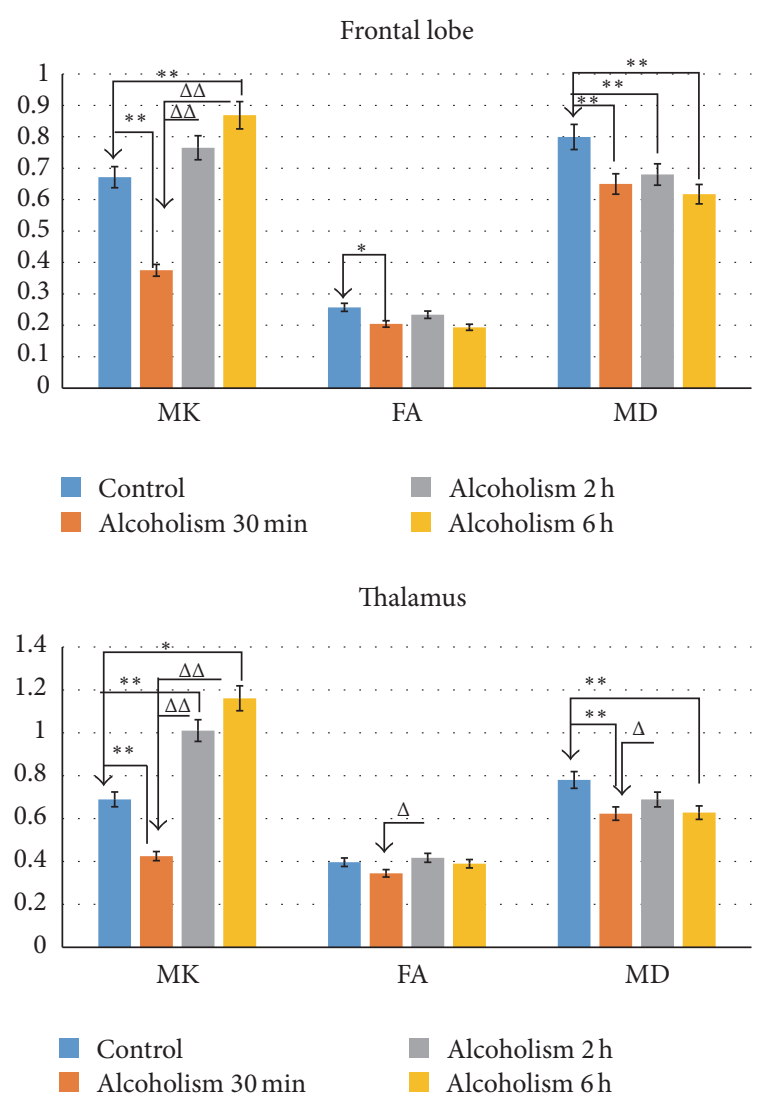

FIgURE 3: Changes in MK, FA, and MD values for frontal lobe and thalamus between controls and EtOH-treated rats. ${ }^{*}$ Statistical significance was based on comparison with controls. ${ }^{\Delta}$ Statistical significance was based on comparison with alcoholism $30 \mathrm{~min}$. ${ }^{*, \Delta} P<0.05$ was considered to indicate a statistically significant difference. ${ }^{* * \Delta \Delta} P<0.01$ was considered to indicate a clear statistically significant difference.

increase in FA and MD was only observed in the thalamus at $2 \mathrm{~h}(p<0.05)$.

No statistically significant difference was observed for the $\mathrm{MK}, \mathrm{FA}$, and MD values between $2 \mathrm{~h}$ and $6 \mathrm{~h}$ after acute alcohol administration.

In the control group, HE staining revealed normal pericapillary spaces and intact blood vessel walls within the frontal lobe and thalamus. In addition, no erythrocytes were observed within the vascular lumina. Neurons exhibited normal morphology with clear round nuclei and a constant nucleus-cytoplasm ratio (Figure 4(a)). For alcoholadministered rats, HE staining mainly revealed neurons with edema and arranged in a disordered pattern (Figure 4(b)).

\section{Discussion}

There was a steady reduction in MD in the frontal lobe and thalamus at each of the observed time points after EtOH administration. This phenomenon has been well documented in our prior investigations $[8,9]$. Changes in $\mathrm{MD}$ value are helpful for distinguishing and identifying the type of edema in lesions. A reduced MD suggests cytotoxic edema, whereas an increased MD indicates vasogenic edema [8, 9, 23]. Therefore, the observed decreased MD value $6 \mathrm{~h}$ after EtOH administration was indicative of cytotoxic edema, which is consistent with our HE staining results.

In contrast, no appreciable change in FA was observed in the frontal lobe and thalamus at the 3 time points except at $30 \mathrm{~min}$ in the frontal lobe. FA, as one of the most important measures calculated from DTI, has been proven susceptible to microstructural changes in white matter integrity [24]. The FA value was thought to decrease as a result of disruption, disorganization, and loss of myelin sheaths and axonal membranes in white matter [11,25-27]. In our study, the temporary progressive decrease in FA throughout the frontal lobe $30 \mathrm{~min}$ after acute alcohol administration may reflect loss of myelin sheaths induced by ethanol, which will be explained later. The lack of change in FA at other times in the frontal lobe and thalamus may reflect the limitation of diffusion tensor imaging.

MK, defined as the average of the kurtosis over all possible diffusion directions, which is the most important measure calculated from the diffusion kurtosis [13]. The change of MK value depends on the structural complexity of the organization. MK decreased in the frontal lobe and thalamus at $30 \mathrm{~min}$, which suggests a reduction in overall diffusional heterogeneity and is likely due to the following three factors: (1) ethanol can cause local cerebral ischemic and hypoxic injury in the early stage, decreasing the activity of $\mathrm{Na}^{+}, \mathrm{K}^{+}$ATPase [9]; at the same time, ethanol disturbs intracellular energy metabolism, increasing the influx of $\mathrm{Ca}^{2+}[28,29]$, which jointly leads to cytotoxic edema; (2) it produces massive oxygen radical, has lipid peroxidation, and damages cell membrane [28]; (3) immunoreaction of the organization exists. All the factors mentioned above collaboratively lead to the demyelination, reduce the limitation of medullary sheath on water molecule, and thus decrease MK value.

Unlike FA and $\mathrm{MD}$, the increased $\mathrm{MK}$ values, which were observed at the $2 \mathrm{~h}$ and $6 \mathrm{~h}$ time points in alcoholadministered rats, demonstrate the sensitivity of MK to subsequent complex microstructural change in response to injury. As the illness develops, myelinoclasis, neuronal necrosis, oxidative stress, secondary gliocyte proliferation, and inflammatory cell infiltration may occur in the diseased areas [30-32], which increased the structural complexity of the organization and induced the increase of MK value.

There are several limitations in this study. First, we only chose the frontal lobe and thalamus as ROIs and did not measure $\mathrm{AK}, \mathrm{RK}, \mathrm{AD}$, and $\mathrm{RD}$. It would be of great interest to examine other regional brain changes including separate brain functional region through DKI metrics. Meantime, we should analyse all DTI and DKI values to figure out more detail of brain microstructure changes after acute alcohol administration. In addition, the sample size used in this study was too small, and we only followed the animals in the acute stage. It would be advantageous to increase the sample size and trace animals through the subacute and chronic phases. Finally, the duration time for the DKI scan was too long. Therefore, future studies will attempt to optimize the scanning parameters. 


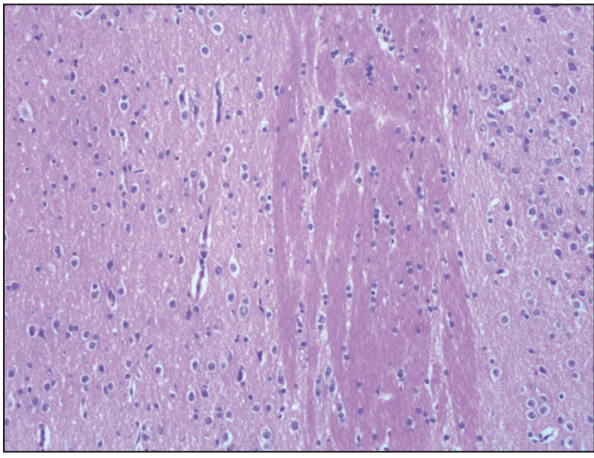

(a)



(b)

FIGURE 4: HE stains of representative acute alcohol intoxication rats and a sham rat. (a) Control rat; (b) acute alcohol intoxication rats at 6 h.

\section{Conclusions}

Compared with DTI, DKI can provide a more comprehensive evaluation of EtOH-related brain changes at varying time points. Vulnerability of the frontal lobe and thalamus to effects of acute alcohol intoxication suggests that changes in DKI of the frontal lobe and thalamus in vivo may be useful in predicting clinical outcome and facilitating early interventions that might reduce more serious sequelae following acute alcoholism.

\section{Competing Interests}

The authors declare that they have no competing interests.

\section{Acknowledgments}

This study was supported by the National Natural Science Foundation of China (Grant no. 81571627), Natural Science Foundation of Guangdong Province, China (Grant no. 2014A030313481), and Shantou University Characteristic Innovation Project of Ordinary University of Guangdong Province, China (Grant nos. 922-38040223 and 92338040404).

\section{References}

[1] S. M. Paul, "Alcohol-sensitive GABA receptors and alcohol antagonists," Proceedings of the National Academy of Sciences of the United States of America, vol. 103, no. 22, pp. 8307-8308, 2006.

[2] C. Sun, L. Shen, X. Li, C. Liu, and Y. Zhou, "Risk of pneumonia in central nervous system injury with alcohol intake: a metaanalysis," International Journal of Clinical and Experimental Medicine, vol. 8, no. 9, pp. 15738-15744, 2015.

[3] M. Field, R. W. Wiers, P. Christiansen, M. T. Fillmore, and J. C. Verster, "Acute alcohol effects on inhibitory control and implicit cognition: implications for loss of control over drinking," Alcoholism: Clinical and Experimental Research, vol. 34, no. 8, pp. 1346-1352, 2010.

[4] W. D. Rooney, J.-H. Lee, X. Li et al., "4.0 T water proton $\mathrm{T} 1$ relaxation times in normal human brain and during acute ethanol intoxication," Alcoholism: Clinical and Experimental Research, vol. 24, no. 6, pp. 830-836, 2000.

[5] J. M. Bjork and J. M. Gilman, "The effects of acute alcohol administration on the human brain: insights from neuroimaging," Neuropharmacology, vol. 84, pp. 101-110, 2014.

[6] M. Bühler and K. Mann, "Alcohol and the human brain: a systematic review of different neuroimaging methods," Alcoholism: Clinical and Experimental Research, vol. 35, no. 10, pp.1771-1793, 2011.

[7] T. Schulte, B. G. Oberlin, D. A. Kareken et al., "How acute and chronic alcohol consumption affects brain networks: insights from multimodal neuroimaging," Alcoholism: Clinical and Experimental Research, vol. 36, no. 12, pp. 2017-2027, 2012.

[8] L. M. Kong, W. B. Zheng, G. P. Lian, and H. D. Zhang, "Acute effects of alcohol on the human brain: diffusion tensor imaging study," American Journal of Neuroradiology, vol. 33, no. 5, pp. 928-934, 2012.

[9] H. Liu, W. Zheng, G. Yan et al., "Acute ethanol-induced changes in edema and metabolite concentrations in rat brain," BioMed Research International, vol. 2014, Article ID 351903, 8 pages, 2014.

[10] E. S. Hui, M. M. Cheung, K. C. Chan, and E. X. Wu, "B-value dependence of DTI quantitation and sensitivity in detecting neural tissue changes," NeuroImage, vol. 49, no. 3, pp. 2366$2374,2010$.

[11] L. Filli, M. Wurnig, D. Nanz, R. Luechinger, D. Kenkel, and A. Boss, "Whole-body diffusion kurtosis imaging: initial experience on non-Gaussian diffusion in various organs," Investigative Radiology, vol. 49, no. 12, pp. 773-778, 2014.

[12] F. Grinberg, E. Farrher, L. Ciobanu, F. Geffroy, D. Le Bihan, and N. J. Shah, "Non-Gaussian diffusion imaging for enhanced contrast of brain tissue affected by ischemic stroke," PLoS ONE, vol. 9, no. 2, Article ID e89225, 2014.

[13] M. Hori, I. Fukunaga, Y. Masutani et al., "Visualizing nonGaussian diffusion: clinical application of q-space imaging and diffusional kurtosis imaging of the brain and spine," Magnetic Resonance in Medical Sciences, vol. 11, no. 4, pp. 221-233, 2012.

[14] J. H. Jensen and J. A. Helpern, "MRI quantification of nonGaussian water diffusion by kurtosis analysis," NMR in Biomedicine, vol. 23, no. 7, pp. 698-710, 2010.

[15] J. H. Jensen, J. A. Helpern, A. Ramani, H. Lu, and K. Kaczynski, "Diffusional kurtosis imaging: the quantification of nonGaussian water diffusion by means of magnetic resonance 
imaging," Magnetic Resonance in Medicine, vol. 53, no. 6, pp. 1432-1440, 2005.

[16] J. A. Stokum, C. Sours, J. Zhuo, R. Kane, K. Shanmuganathan, and R. P. Gullapalli, "A longitudinal evaluation of diffusion kurtosis imaging in patients with mild traumatic brain injury," Brain Injury, vol. 29, no. 1, pp. 47-57, 2015.

[17] R. T. Khisti, M. J. VanDoren, T. O’Buckley, and A. L. Morrow, "Neuroactive steroid $3 \alpha$-hydroxy- $5 \alpha$-pregnan-20-one modulates ethanol-induced loss of righting reflex in rats," Brain Research, vol. 980, no. 2, pp. 255-265, 2003.

[18] K. Kamagata, H. Tomiyama, T. Hatano et al., "A preliminary diffusional kurtosis imaging study of Parkinson disease: comparison with conventional diffusion tensor imaging," Neuroradiology, vol. 56, no. 3, pp. 251-258, 2014.

[19] K. Kamagata, H. Tomiyama, Y. Motoi et al., "Diffusional kurtosis imaging of cingulate fibers in Parkinson disease: comparison with conventional diffusion tensor imaging," Magnetic Resonance Imaging, vol. 31, no. 9, pp. 1501-1506, 2013.

[20] H. F. Moselhy, G. Georgiou, and A. Kahn, "Frontal lobe changes in alcoholism: a review of the literature," Alcohol and Alcoholism, vol. 36, no. 5, pp. 357-368, 2001.

[21] S. Ide, S. Kakeda, and Y. Korogi, "Anatomy of the thalamus," Brain and Nerve, vol. 67, no. 12, pp. 1459-1469, 2015.

[22] J. F. A. Poulet, L. M. J. Fernandez, S. Crochet, and C. C. H. Petersen, "Thalamic control of cortical states," Nature Neuroscience, vol. 15, no. 3, pp. 370-372, 2012.

[23] I. Cernak, R. Vink, D. N. Zapple et al., "The pathobiology of moderate diffuse traumatic brain injury as identified using a new experimental model of injury in rats," Neurobiology of Disease, vol. 17, no. 1, pp. 29-43, 2004.

[24] P. Mukherjee and R. C. McKinstry, "Diffusion tensor imaging and tractography of human brain development," Neuroimaging Clinics of North America, vol. 16, no. 1, pp. 19-43, 2006.

[25] P. J. Basser, "Inferring microstructural features and the physiological state of tissues from diffusion-weighted images," NMR in Biomedicine, vol. 8, no. 7-8, pp. 333-344, 1995.

[26] C. B. Fortier, E. C. Leritz, D. H. Salat et al., "Widespread effects of alcohol on white matter microstructure," Alcoholism: Clinical and Experimental Research, vol. 38, no. 12, pp. 2925-2933, 2014.

[27] E. J. Grossman, J. H. Jensen, J. S. Babb et al., "Cognitive impairment in mild traumatic brain injury: a longitudinal diffusional kurtosis and perfusion imaging study," American Journal of Neuroradiology, vol. 34, no. 5, pp. 951-957, 2013.

[28] H. W. Sippel, "The acetaldehyde content in rat brain during ethanol metabolism," Journal of Neurochemistry, vol. 23, no. 2, pp. 451-452, 1974.

[29] B. Tabakoff, R. A. Anderson, and R. F. Ritzmann, "Brain acetaldehyde after ethanol administration," Biochemical Pharmacology, vol. 25, no. 11, pp. 1305-1309, 1976.

[30] C. J. Kane and P. D. Drew, "Inflammatory responses to alcohol in the CNS: nuclear receptors as potential therapeutics for alcoholinduced neuropathologies," Journal of Leukocyte Biology, vol. 100, no. 5, pp. 951-959, 2016.

[31] F. T. Crews and K. Nixon, "Mechanisms of neurodegeneration and regeneration in alcoholism," Alcohol and Alcoholism, vol. 44, no. 2, pp. 115-127, 2009.

[32] F. Crews, K. Nixon, D. Kim et al., "BHT blocks NF- $\kappa$ B activation and ethanol-induced brain damage," Alcoholism: Clinical and Experimental Research, vol. 30, no. 11, pp. 1938-1949, 2006. 


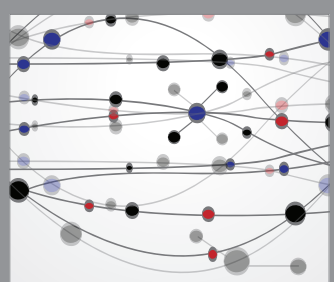

The Scientific World Journal
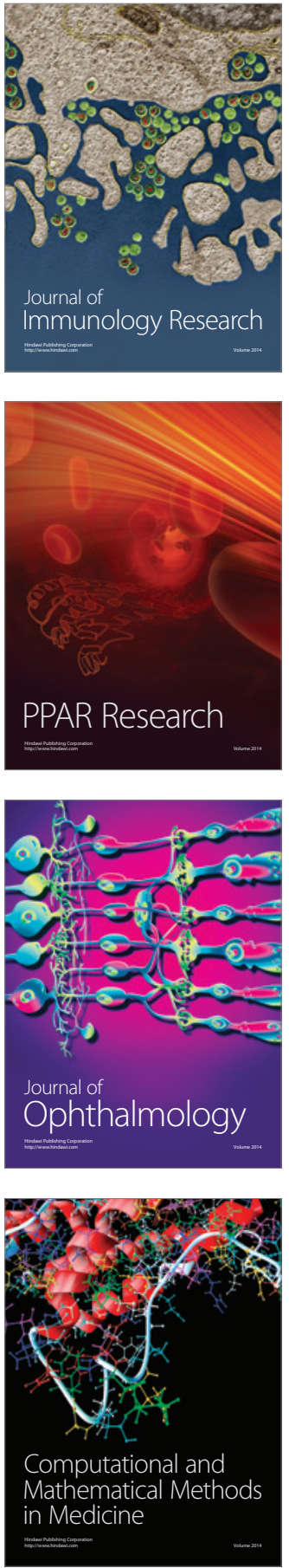

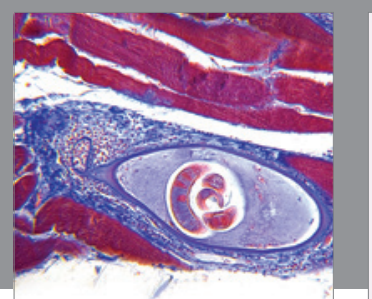

Gastroenterology Research and Practice
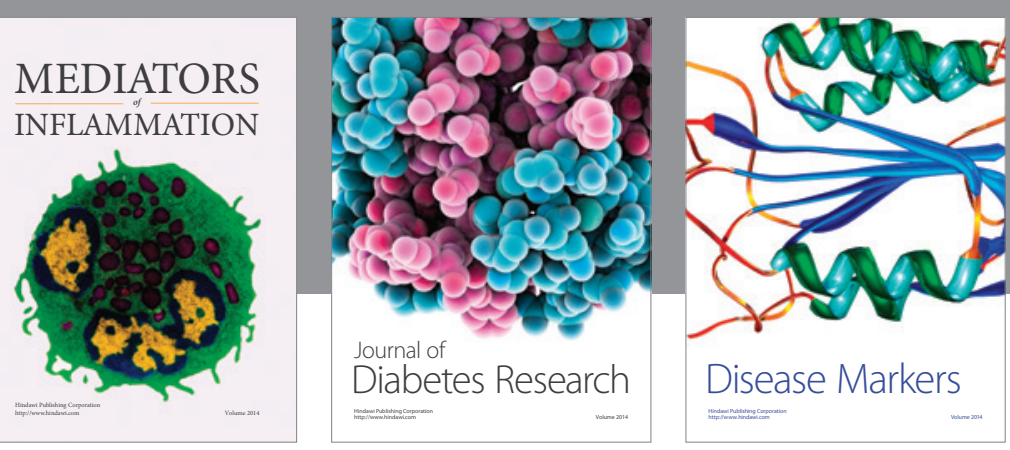

Disease Markers

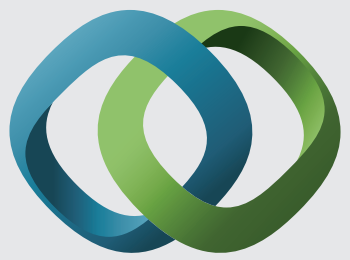

\section{Hindawi}

Submit your manuscripts at

https://www.hindawi.com
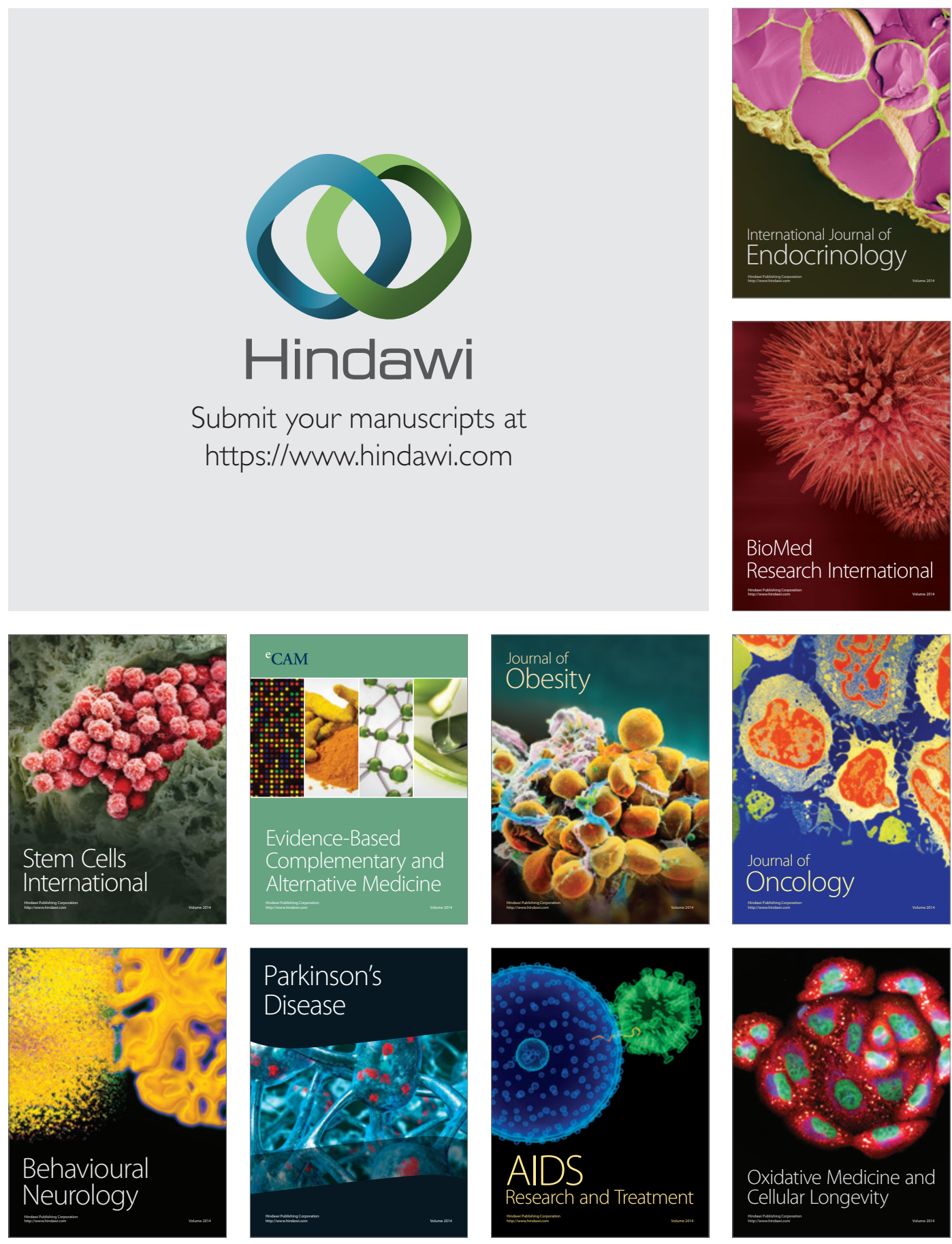